\title{
Contents
}

Deep Pool Immersion Therapy

National Council for the Care of Cripples in South Africa

Some Impressions of Rehabilitation in U.S.A. and Canada

Group Demonstration in Cape Town Post Graduation Course

General

Branch News

Students' Column

National Fitmess Congress

Vacancies

$\begin{array}{cccccc} & & & & & \text { Page } \\ \ldots & \ldots & \ldots & \ldots & \ldots & 3 \\ \ldots & \ldots & \ldots & \ldots & \ldots & 6 \\ \ldots & \ldots & \ldots & \ldots & \ldots & 7 \\ \ldots & \ldots & \ldots & \ldots & \ldots & 9 \\ \ldots & \ldots & \ldots & \ldots & \ldots & 10 \\ \ldots & \ldots & \ldots & \ldots & \ldots & 11 \\ \ldots & \ldots & \ldots & \ldots & \ldots & 12 \\ \ldots & \ldots & \ldots & \ldots & \ldots & 14 \\ \ldots & \ldots & \ldots & \ldots & \ldots & 16\end{array}$

\section{DEEP POOL IMMERSION THERAPY}

\author{
Mr. K. I. S. NICOL, M.S.A.S.P., M.C.S.P., H.T., D.P.E \\ Paper delivered to the Post-Graduate Course in Cape Town, 1961
}

Hydrotherapy has developed as a branch of physical medicine, from the use of natural waters as a means of internal and external treatment. Hydrotherapy being at section of spa therapy, may be sub-divided into many parts of wet therapy. The whole of this embraces a field of sufficient importance to justify a post-registration qualification for Chartered Physiotherapists in Great Britain, who direct it to their patients.

\section{Deep Pool Therapy}

Deep-pool immersion therapy being a part of hydrotherapeutic measures now available, is no doubt, worthy of critical evaluation. Although deep pools are often expensive to instal, and to maintain, more are at present being planned or built. There is nothing new or mysterious about the beneficial effects of pool-therapy, the Romans used it extensively. However, the techniques have been brought up to date so that it is specialised.

The growing interest of the medical profession, especially that of the Orthopaedic Surgeons, have been quick to take advantage of properly applied pool therapy, particularly in respect 10 muscle and joint re-education, as a means of speeding up convalescence. The ideal medium before permitting weight bearing, is to administer controlled aclivity and specific manipulations in water, in order to promote muscle development with an efficient circulatory function. It can be envisaged that the increasing demand will continue to grow for such treatment, embracing a great variety of conditions.

\section{Water}

\section{Equipment}

The water may be mineralized or plain. Natural waters from springs do not need chlorinating, if kept constantly flowing. So long as it is devoid of taste, smell and does not stain, it is economical.

\section{Temperature}

The temperature ranges from $90^{\circ} \mathrm{F}$. to $102^{\prime} \mathrm{F}$. averaging $96^{\circ} \mathrm{F}$. An under water douche if used for massage being $5^{\circ}-10^{\circ} \mathrm{F}$. above the temperature of the pool, and is connected to a hot and cold mixing chamber.

Size

The size and shape of pools vary, with the depth from $2^{\prime} 6^{\prime \prime}$ to $5^{\prime} 6^{\prime \prime}$ averaging $4^{\prime} 6^{\prime \prime}$ for adults. It should be surfaced with non-slip tiles and under these a special lining of lead or asphalt to prevent leakage. The capacity is from 600 gallons to 10,000 gallon, 6,000 gallons being a good average.

Colour decoration, lighting and ventilation should receive consideration for congenial surroundings and to prevent condensation.

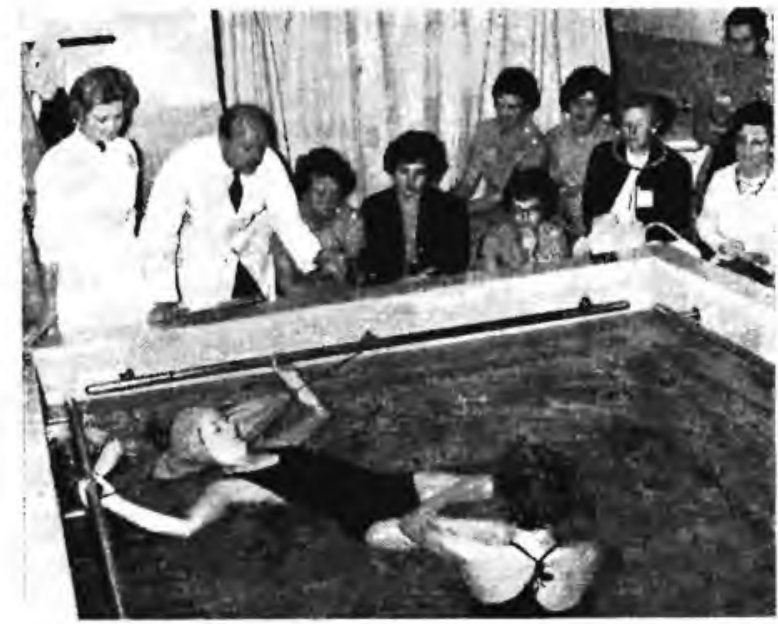

Physiotherapists attending a two-day refresher course at the University of Cape Town were given a demonstration of the technique of suspension at the Princess Alice Home, Retreat, recently. Instructing is Mr. K. Nicol (in white coat), and looking on is Mrs. G. Steyn (left) the home's head physiotherapist. (Acknowledgement to Argus.)

\section{Pool Apparantus}

Where patients ale severely handicapped, they are lowered into the pool on a "gantry", a series of slings or a stretcher, securing the patient by straps. The gantry is run out over the pool and let down by some hydraulic mechanism.

The patient may be treated in the stretcher or canvas slings so long as this does not impede the exercise of the part under treatment.

An adjustable inclined plinth, attached to one side of the pool is also useful in the early stages for specific purposes, e.g. to localise movements and manipulations.

Most of the apparatus should be taken out to drain overnight, to offset deterioration.

As the force of gravity is reduced in water, the patient may be supported in the upright position, by parallel bars running across the pool. Weighted and moveable stools of benches are useful to exercise in sitting, also in standing for children. 
There is a handrail all round at water level to provide hand and foot fixation and to serve as a headrest in the corners along with the use of a sling in suspension. To assist body buoyancy, air rings and cork floats are used. For re-education of walking, splints of perspex or fibre glass etc. help to stabilise the legs.

lt is desirable to use the minimum of apparatus, by making full use of the supporting water only.

\section{Advantages of Pool Therapy}

Although some hydrotherapeutic principles can not be applied so well, if at all, by any other method, it can not produce a maximum effect in many cases, without the simultaneous use of various dry treatments on alternate days. Daily use of pool treatments can become exhausting for certain patients.

(a) Because of the large limb and body movements permissible with buoyancy assisting in a pool, the patient experiences a feeling of well being, and of enjoyable achievement in even the simplest of manoeuvres.

(b) It is in this water medium that all paresed muscles may accomplish their initial joint movements, especially to master weight-bearing, static sitting and walking, long before this is performed on land.

(c) Even resistance exercises in many cases can only be performed in water when considering progression to the pro-prioceptive neuro-muscular facilitation technique out of water. This is a direct advantage over its sister suspension method of sling therapy, in the department, also in respect of improving body balance in all positions, along with the addition of its complete thermal accompaniment.

Here is a great opportunity to develop muscles, both intrinsically and extrinsically, improving neuro-muscular co-ordination and stablisation for functional activity.

\section{Therapeutic Effects}

The main effect is the force of buoyancy which is of greater use in respect of graduated therapy, as compared with the effect of gravity being eliminated.

Friction is to all intents and purposes, eliminated in the water due to absence of weight on the limb, allowing passive and active movement through an increased range, along with reduced pain.

The thermal effect of the surrounding water warms the total surface area of the body (excluding the head), inducing relaxation of muscle tissue giving a sedative effect on nerve endings, with improved circulation, thus enhancing a more efficient muscle function.

The physiological function is assisted by improved metabolism, skin activity and of the heat regulating mechanism. The benefit to be gained is most marked in all cases of muscle re-education which is of primary importance, improving power and control.

The under-water douche, produces a hyperaemia around the joints, and assists in the adsorption of inflammatory and fibrous thickenings, dispersing them in muscles, around tendons and joints. Thus it offsets the development of traumatic arthritis in some fracture and post-operative orthopaedic cases. It also has a slight analgesic effect and helps in overcoming the discomforts caused by stretching the tissues. The thermal effect assists in the removal of waste products due to faulty metabolism, accelerating the interchange, between blood and tissue fluids by activating the circulation of blood and lymph.

The psychological benefit is obvious while handling a patient in water, gaining confidence in his treatment, generally, leading to increased perseverance to progress and achieve further goals.

\section{Considerations}

Many patients have superimposed psychological causations. Here $\mathrm{I}$ venture to suggest that over $70 \%$ of conditions we treat are psychosomatic in origin, so it is desirable to remove the patients from the influences which may prevail in their working and domestic life, wherever possible, hence the planning of large hospitalised hydrotherapy centres, especially for rheumatic cases. The patient is more easily able to prove his capabilities assisted by the skilled hydrotherapist.

\section{Hydrostatic Pressure}

Hydrostatic pressure-is exerted equally in all directions and increases roughly by $\mathbf{l} \mathrm{lb}$. per square inch for every two feet vertically below the surface. This pressure has a marked effect on respiration and circulation, so that time must be allowed for adjustment and the amount of immersion of the thorax will vary with different types of cases. It is this hydrostatic pressure that enables a patient to stand and walk in water, long before he can do it on land.lt acts as a corset around the body, giving the abdominal muscles support: This enables the diaphragm to work easily, aiding the venous return as the breathing is deeper and vital capacity increased.

\section{Dynamic Pressure}

Dynamic pressure-is utilised to vary the resistance of movements. This is increased if the speed of the movement is accelerated. By relationship of the body to the water, the greater the surface area of the body the greater the resistance and difficulty for the water to flow past it. Therefore movements and positions are varied accordingly. This calls on the therapists ingenuity.

Wave resistance-is considered with weak patients as an initial progression.

\section{Buoyancy}

Buoyancy - the upthrust of the water is utilised to first of all support the patient, as in a continuous sling, to assist and to resist body movements according to the muscle strength.

Atmospheric temperature and humidity--this hot and humid environment will retard heat loss from the body. It is very necessary to give attention to signs of fatigue as heat is generated from muscular activity. Therefore the body should not be continually immersed up to the neck, and the duration of treatment must be regulated accordingly and consideration should be given to daily pool therapy in this respect.

In view of the fact that it is physically arduous to work immersed in warm water, there should be ample physiotherapists to assure a rotation of those working in a busy deep pool centre.

\section{Differences}

Compared with other forms of physical treatment in the physiotherapy department, the difference is obvious; a wet treatment being administered with total thermal application in suspension, utilising buoyancy as a reverse to the effect of gravity. Along with this, humidity, hydrostatic and dynamic pressures are given consideration when perfecting the technique of pool therapy.

In the pool, patterns and specific movements are initiated with greater active work. It is instinctive to perform some movement with enjoyment in water and so develop an ability consciousness which is not always considered by the patient on dry land, while lying in apathy on a bed.

The dry form of therapy most similar to that in the pool is kinesiotherapy in sling suspension. Although slings are less expensive to install it does not suffice or is it so exacting for the physiotherapist, especially in the early stages of a treatment programme. However, pool therapy should be correlated with appropriate treatment in the department, and need not be used if the latter is all that is necessary.

In water a greater number of synergist acting muscles are brought into play because of the instability of a water medium. This in turn facilitates involuntary activity of intrinsic muscle groups when they are incapable of performing as prime movers. Hence balance and swimming exercises in various positions will strengthen these and the important large group trunk stabilisers.

\section{Types of Conditions Treated}

The aim of the two main groups of conditions treated in the deep immersion pool are:

(a) Restoration of skeletal muscle strength.

(b) Improved range of joint function. 
In the former, the following types of conditions are conIn the acute anterior poliomyelitis, flaccid paralyses, sidered. some spastic hemiplegia, muscular dystrophies, debility and where inter-thoracic vital capacity needs to be improved in where iratory involvement.

Secondly, for increasing joint mobility, pool therapy is used to treat the effects of traumatic joint injuries, sprains and fractures, expecially those following industrial injuries and stiffness following immobilization of joints. Periarthritis, fibrositis, chronic osteo and rheumatoid arthritis, spondylitis, the followup after certain post-operative conditions and orthopaedic procedures of large joints, also to offset the development of osteoporosis.

\section{Contra-indications}

1. Respiratory disorders that are sufficiently severe to be a danger to the patient's recovery, especially if the abdominals are also badly affected causing difficulty to expel water from the throat.

2. Inflammatory conditions in acute and sub-acute synovitis, tuberculosis, arthritis, febrile cases, and infective skin conditions.

3. In muscular dystrophy, if there is difficulty with swallowing and some inability to hold the head upright, is an additional contra-indication.

4. Cardiac involvements, high blood pressure and arterioclerosis; renal diseases, if cystitis, nephritis or incontinence are present, pool treatment should be postponed.

\section{Technique of Treatments}

Floating - it is advantageous for the patient to be taught floating positions first, for its psychological importance and to obtain muscle relaxation. When apprehensive, the body assumes the attitude of flexion but floating supine counteracts this tendency, especially if the patient abducts the arms and legs, retracting the shoulder blades together.

To prevent the legs from sinking, the abdominals are contracted slightly. Progress to floating in the prone position, giving less manual support so that the patient performs the necessary movements himself to improve balance contro by group muscle action.

A]though it is more in keeping to perform all movements, with the limbs immersed, I personally would agree to a patient swinging an arm or leg out of the water, because it demonstrates the patient's capability of that particular movement, and it is often psychologically beneficial, therefore, not to be restrained unduly.

This leads me to the proprioceptive neuromuscular facilitation technique, being initiated in water to facilitate progression, later exercising this method in the physiotherapy department, this being a direct up-grading of the patient's therapeutic achievements.

Group Pool Therapy-Individual pool work is progressed to Group work to stimulate the competitive element and to appreciate the effect of space, for freedom of movement. In this instance specific and general active remedial exercises are carried out to include walking, swimming and playing games.

Under water exercises are not merely doing the same dry land exercises, only in water.

The application is a special technique and must be studied and directed to obtain the proper progressions and use the water medium to the full advantage, so obtaining the expected results.

Progression-It is well worth while that the therapist should try all movements in water and in different positions to obtain a comprehensive understanding of the effect of exercises. For instance the muscle work of walking is in reverse of that on land. Buoyancy assists the legs to come up and resisting buoyancy when placing the leg down, instead of it being assisted by gravity.

Movements in water are either Passive, Assisted, Active or Resisted.

\section{Ten-point Progression of Hydrotherapeutic Muscle Re-education}

I. Purposeful passive movements. (To re-establish a pattern of movement).

2. Static holding contractions, with the limb at buoyant level.

3. Active buoyant-assisted movements are performed.

4. Lateral unassisted movements against the viscosity of the water, with the limb at buoyant level.

At this stage, it can be observed that the buoyant level will be raised as the muscle power improves, before it is possible to detect such improvement with manual muscle testing.

5. At surface level apply wave resistance.

6. Movement with buoyancy resisting.

7. Reduced manual and artificial support.

8. Simultaneous contraction with both origin and insertion of a muscle.

9. Increased speed of limb movement.

10. Under water manual resistance utilising the proprioceptive neuromuscular facilitation technique. This includes stabilization and balance training in the floating and kneeling positions as done on a mat, also in sitting and standing.

\section{Conclusion}

While considering pool therapy it is stressed that hydrotherapy is only a part of the physical medicine armamentarium which is used conjointly with other measures in the physiotherapy department. It is a method of improving conditions with remarkable rapidity, treating the patient as a whole while concentrating on specific improvements.

It is ideal for a physiotherapist trained in hydrotherapy to follow a patient's treatment regime through from the pool to the department, being the therapist most familiar with the patient's capabilities.

The beneficial results obtained in suitable cases leads one to suppose that a wide use of this form of rehabilitation is long overdue, throughout physiotherapeutic centres, which will become more important in the future.

In South Africa we have a few hospitals with deep pool installations, but there are also numerous underdeveloped places from which natural mineralized springs flow. These may well be developed into spas and the South African Society of Physiotherapy may well contemplate making the necessary approach to develop these into useful rehabilitation centres on a National scale.

There appears to be a scarcity of physiotherapists trained in Hydrotherapy, so it is heartening to see that efforts are being made to introduce students of physiotherapy in deep pool immersion techniques.

\section{REFERENCES}

T. G. Reah, 1957, The British Journal of Physical Medicine, 20,1 .

W. Menzies, 1950, Physiotherapy Journal, 36, 9.

\section{SOME EXAMINATION HOWLERS Terminology}

Physiotherapy: provides support for stretched ligaments.

Frictions: are given for hyperaemia. When hyperaemia is sufficient the patient moves to a chair.

The Head: is extremely vital.

Massage: the tissues are picked off the bone.

Muscle: Extensor digitorum communist.

Frozen Shoulder: The arm was taken up by the elevator. 